OPEN ACCESS

Edited by:

Wing Chee So,

The Chinese University of

Hong Kong, Hong Kong

Reviewed by:

Ming Lui,

Hong Kong Baptist University,

Hong Kong

John-John Cabibihan,

Qatar University, Qatar

*Correspondence:

Yosuke Kita

kitay@ncnp.go.jp

Specialty section: This article was submitted to

Special Educational Needs,

a section of the journal

Frontiers in Education

Received: 14 April 2017 Accepted: 11 September 2017 Published: 27 September 2017

Citation:

Kita Y, Gunji A, Sakuma R, Goto T, Okumura Y and Inagaki M (2017) Training Attention to the Other's Need Improves Helping Behaviors in Children with Autism Spectrum

Disorder: A Behavioral Analysis Using a Two-dimensional Motion Capture System.

Front. Educ. 2:50

doi: 10.3389/feduc.2017.00050

\section{Training Attention to the Other's Need Improves Helping Behaviors in Children with Autism Spectrum Disorder: A Behavioral Analysis Using a Two-dimensional Motion Capture System}

\author{
Yosuke Kita ${ }^{1 *}$, Atsuko Gunji 1,2, Ryusuke Sakuma1,3, Takaaki Goto ${ }^{1,2}$, Yasuko Okumura \\ and Masumi Inagaki ${ }^{1}$
}

'Department of Developmental Disorders, National Institute of Mental Health, National Center of Neurology and Psychiatry (NCNP), Tokyo, Japan, ${ }^{2}$ College of Education, Yokohama National University, Yokohama, Japan, ${ }^{3}$ Graduate School of Liberal Arts, Shirayuri College, Tokyo, Japan

While children with autism spectrum disorder (ASD) can acquire helping behaviors through appropriate interventions, changes in behaviors prior to helping (pre-helping behaviors) remain unclear. In the present study, we examined the effects of social skills training (SST) on helping and pre-helping behaviors in two children with ASD by using a two-dimensional motion capture system. During the SST, the children learned one helping behavior that they lent their items to their partners, and we measured their head movements before initiating the helping behavior (i.e., pre-helping behavior). As a result of SST, the participants became able to help others in response to less-explicit social stimuli after the intervention. Regarding pre-helping behaviors, the children with ASD before the intervention looked straight at the helpee (i.e., recipient of the help) more often than did typically developing peers, and such a behavior was shown to increase after SST. These results indicate that although spontaneous attention to social stimuli may be reduced in children with ASD, success in attending to a helpee could lead to the emergence of helping behaviors. Moreover, the changes in pre-helping behavior indicate an increase in children's attention to the helpee after the intervention, which may have enhanced their sensitivity to persons in need.

Keywords: autism spectrum disorders, social skills training, helping behavior, motion capture, educational intervention research

\section{INTRODUCTION}

Children with autism spectrum disorder (ASD) have difficulties in social interactions (Tsujii, 2004), as reflected by scarce prosocial behaviors (Carter et al., 2005). Prosocial behaviors are defined as voluntary behaviors intended to benefit other individuals (Eisenberg, 1982), such as altruistic and helping behavior, and the latter is particularly effective for developing relationships with others 
(Harris et al., 1990). Therefore, helping behaviors in children with ASD and intervention approaches to facilitate them have been considered in a number of studies.

Previously, helping behaviors in ASD have been shown to increase after interventions that set them as target behaviors. In a study by Matsuoka et al. (1999), the use of video-monitoring and role-reversal methods made it possible for a young adult with ASD to perform and adjust (i.e., not helping when helping was unnecessary) helping behaviors for others in a difficult situation. Similar results have been reported in elementary-to-junior high-school students with ASD (Matsuoka and Noro, 2001; Suto, 2006; Suto and Oishi, 2007). These successful outcomes may be due to an improvement in attention toward social stimuli, such as the words of others and situations in which they were placed (Suto, 2008). Based on these findings, shaping and adjustment of helping behaviors in ASD can be achieved through interventions that directly target such behaviors.

While previous studies have primarily focused on shaping helping behaviors, little attention has been paid to the actions prior to helping (i.e., pre-helping behaviors) or the effect of interventions on them (Sugimura, 2009). According to Eisenberg (1986), the emergence of helping behaviors involves three phases: attention to the other's need, motivation, and linkage between intention and behavior. Given the well-known difficulties of children with ASD in understanding emotions (Bacon et al., 1998; Downs and Smith, 2004; Liebal et al., 2008) and theory of mind (Sally and Hill, 2006), their problems with helping behaviors may stem from the attention and/or motivation phases (Eisenberg, 1986) that precede the performance of helping behavior (Sugimura, 2009).

Therefore, the present study aimed to examine the effects of social skills training (SST) on helping and pre-helping behaviors in children with ASD. Among the phases proposed by Eisenberg (1986), we focused on attention to the other's need and examined differences between children with ASD before the intervention and typically developing children, as well as intervention-related changes in those with ASD. This investigation should contribute to the further development of intervention approaches to facilitate their social interactions.

\section{METHODS}

\section{Participants}

The participants with ASD were two boys in the first and second grades (Child A: aged 6 years 2 months; Child B: aged 7 years 10 months, Table 1) who had been diagnosed by more than one pediatric neurologist (American Psychiatric Association, 2013). They showed no physical or language problems from birth to infancy and had no history of other neurological or psychiatric disorders. Their chief complaints were severe difficulties in having conversations and building relationships with other children due to lack of interest. As shown in Table 1, they had normal intellectual abilities and were not medicated. The participants and their guardians were recruited by their attending physician to take part in an SST group after providing a detailed explanation regarding participation. The inclusion criteria for the SST participants were (1) being first or second graders at elementary schools, as they have more opportunities to act in a group, and
TABLE 1 | Profiles of participants with ASD.

\begin{tabular}{|c|c|c|c|}
\hline & & Child A & Child B \\
\hline \multicolumn{2}{|l|}{ Sex } & Male & Male \\
\hline \multicolumn{2}{|c|}{ Age (years:months) } & $6: 02$ & $7: 10$ \\
\hline \multicolumn{2}{|c|}{$\begin{array}{l}\text { Age with confirmed diagnosis } \\
\text { (years:months) }\end{array}$} & $5: 04$ & 5:03 \\
\hline \multicolumn{2}{|c|}{ Comorbidity } & None & None \\
\hline \multicolumn{2}{|c|}{ Medication before and during SST } & None & None \\
\hline \multirow[t]{3}{*}{ WISC-III } & $\mathrm{FIQ}$ & 90 & 108 \\
\hline & VIQ & 94 & 110 \\
\hline & $\mathrm{PIQ}$ & 89 & 104 \\
\hline \multirow[t]{2}{*}{ PARS } & Infant & 15 & 37 \\
\hline & Present & 24 & 21 \\
\hline
\end{tabular}

ASD, autism spectrum disorder; SST, social skills training; WISC-III, Wechsler Intelligence Scale for Children-Third edition; FIQ, full-scale IQ; VIQ, verbal IQ; PIQ, performance IQ; PARS, Pervasive Developmental Disorders Autism Society Japan Rating Scale (cutoff points: infant $>$ 5, present $>7$ ).

(2) complaining of difficulties in making friends and taking part in group activities with similar-aged peers. Exclusion criteria were (1) apparent neurological deficit(s), (2) moderate-to-severe intellectual disability, and (3) pronounced hyperactivity/impulsivity. Child A and Child B satisfied all of the criteria.

In addition, four typically developing boys participated in this study as a control group (first graders, aged 6 years 10 months to 7 years 3 months), who had no neurological or psychiatric disorders including ASD. They did not take part in SST and joined only a separate experimental session. Prior to participation, written informed consent was obtained from all ASD and typically developing children as well as their guardians after a full explanation of the study protocol, which was in accordance with the Declaration of Helsinki and approved by the ethics committee at the National Center of Neurology and Psychiatry (22-4-JI14).

\section{Intervention Framework}

Short-term intensive SST was conducted for a month, consisting of 12 sessions ( $3 \mathrm{~h}$ each), in a group setting with 10 participants: 2 children with ASD, 1 child with attention-deficit hyperactivity disorder, 3 instructors paired up with the children, 1 instructor taking charge of the entire group, and 3 assistant instructors. Each session of SST proceeded as follows: (1) opening of session (approximately $15 \mathrm{~min}$ ), (2) individual study time (45 min), (3) activity 1 (45 min), (4) rest (15 $\mathrm{min}),(5)$ activity 2 (45 $\mathrm{min}$ ), and (6) closing of session (15 min). The 12 sessions were classified into pre-baseline period (sessions 1-3), instruction period 1 (sessions 4-6), midterm baseline period (session 7), instruction period 2 (sessions 8-10), and post-baseline period (sessions 11-12). None of the typically developing children participated in SST, and they only completed one experimental session without individual study time.

\section{Intervention Procedure}

The target behavior of SST was set as helping, which was defined as children lending their own stationery to an instructor with whom they worked in pairs during the preparation for activity 1 or 2. Participants learned the target behavior by watching model movies and answering follow-up questions during the 
individual study time in each session. The movies showed a scene with two persons preparing for activities 1 and 2. One of them (P1) had all of the necessary things, while the other (P2) did not; therefore, P2 was unable to join the activity. At this point, the instructor stopped the movie and asked the children questions regarding the scene (e.g., What is happening here?), P2's situation (e.g., What is wrong with P2?), P2's mental state (e.g., What do you think P2 is feeling?), and the behavior that was expected from P1 (e.g., What should P1 do?). Questions were repeated with additional instructions until the children gave appropriate answers, which were followed by the presentation of the remaining movie depicting the correct answer (i.e., P1 lending his/her stationery to P2).

Subsequently, preparations for activities 1 and 2 started, during which the instructor in the pair intentionally lacked a necessary item. If the children noticed this issue and lent their stationery to the instructor, this was recognized as successful helping behavior and was reinforced by praise from the instructor. To reduce habituation effects, situations in which the instructor required and did not require help were randomly assigned to activities 1 and 2 in each session.

Learning via the model movie was administered only in instruction periods 1 and 2 , and not in the pre-baseline, midterm baseline, or post-baseline periods, which resulted in an experimental design A-B-A-B-A (e.g., Wincze et al., 1972).

\section{Evaluation of Helping Behavior}

Helping behaviors in children were evaluated based on the explicitness of the social stimuli that served as prompts for such behavior. These prompts were categorized in four levels, defined by the degree of behavioral and/or verbal stimulation provided by the instructor (Figure 1A). At level 1, the instructors looked around for the necessary item without using verbal stimulation.
Levels 2-4 involved using weak (e.g., "uh-oh"), moderate [e.g., "Oh, __ is missing..." (mutter to oneself)], and strong verbal stimulation [e.g., "Hey, I don't have a__" (talk to the children)], respectively, while looking around for the thing. It was expected that prompts of higher levels would capture children's attention more easily than would those of lower levels.

Prompts of each level were attempted a maximum of $4 \times(10 \mathrm{~s}$ per attempt). If the helping behavior did not occur, the instructor moved on to prompts of the next level (maximum attempts: 4 levels $\times 4$ attempts $=16$ ). If the helping behavior occurred, no further prompts were used and the instructor continued the preparations for the activities. The level of prompt and number of attempts made before each helping behavior were recorded by the instructor and checked by other researchers via digital video recordings of the sessions. This evaluation was conducted in all sessions from pre-baseline to post-baseline periods for the children with ASD, while the typically developing children went through the same procedure in a separate experimental session.

\section{Evaluation of Pre-Helping Behaviors}

For the examination of pre-helping behaviors, we used a twodimensional motion capture system (Figure 2). Prior to each session, the children and instructors were asked to wear a hat with markers in different colors (Figure 2A), which were tracked by four digital video cameras installed on the ceiling (arranged at the four corners of the room, fisheye lens, 15 frames per second) to record their movements during the session from a bird's-eye view (Figure 2B). The video recordings were then used to obtain their positional information on a two-dimensional surface (Figure 2C). These procedures were carried out using a Kinema recorder and tracer (Kissei Comtec Co., Ltd., Japan).

In the two-dimensional surface (i.e., $x y$ coordinate system), we let $x$ be the relative location of the instructor with respect to the child's
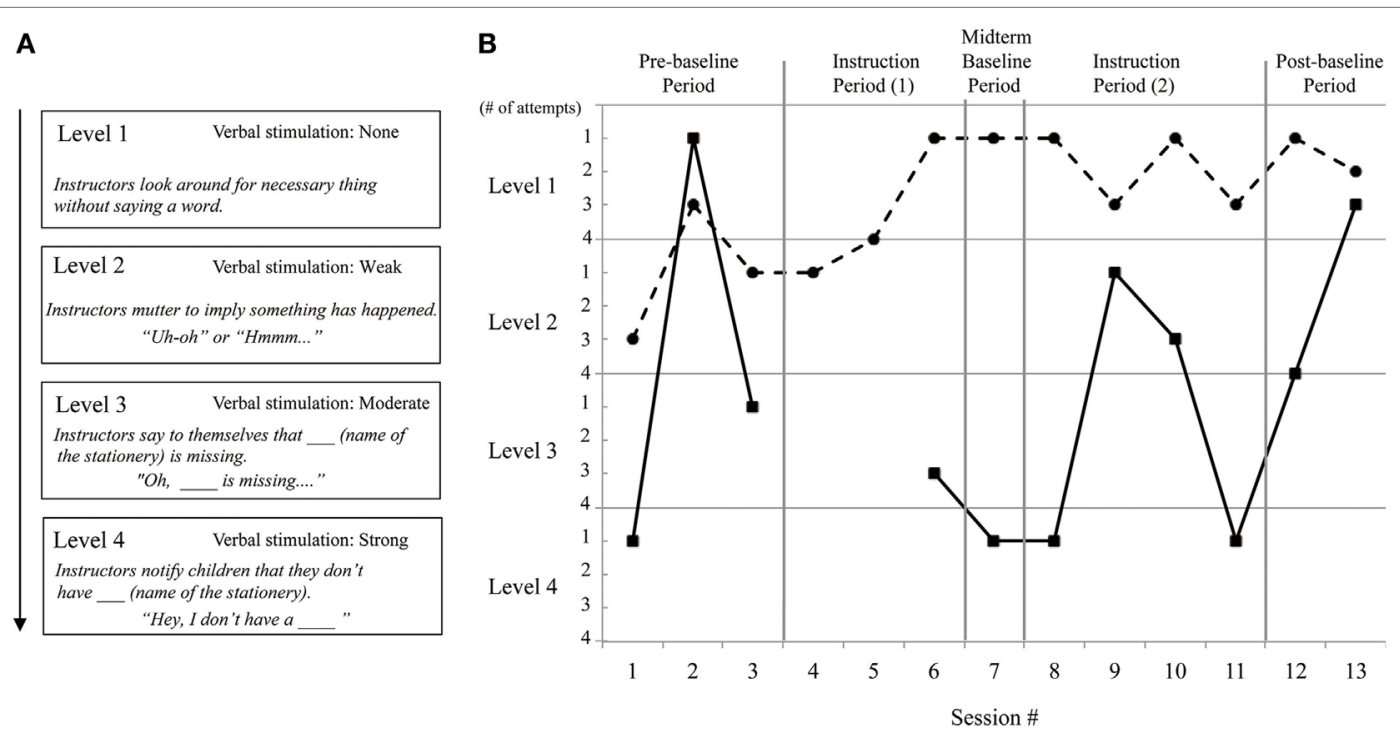

FIGURE 1 | (A) Levels of prompts for helping behavior and descriptions. Four attempts were made at each level. If no helping behavior occurred, the instructor moved on to the next level. (B) Levels of prompts and numbers of attempts required before the children with ASD helped their instructor. Solid line: Child A; dashed line: Child B. 
A

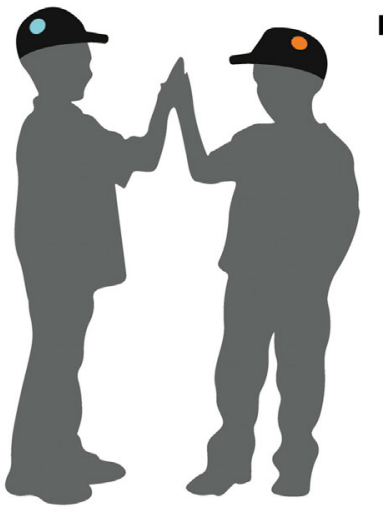

B

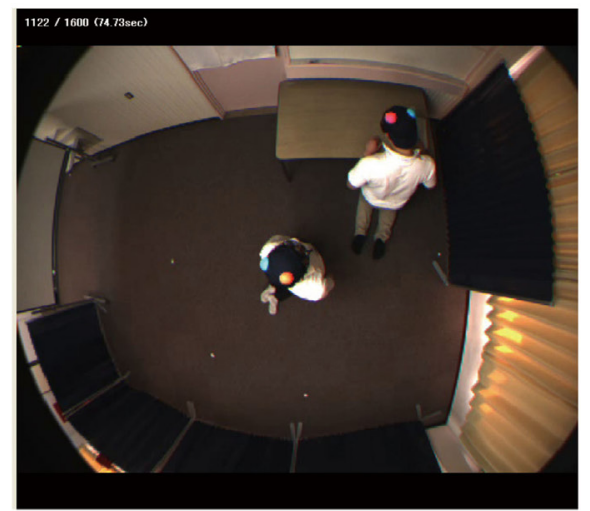

C

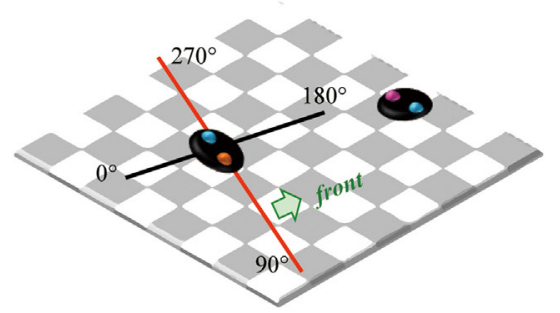

D

$\mathbf{E}$

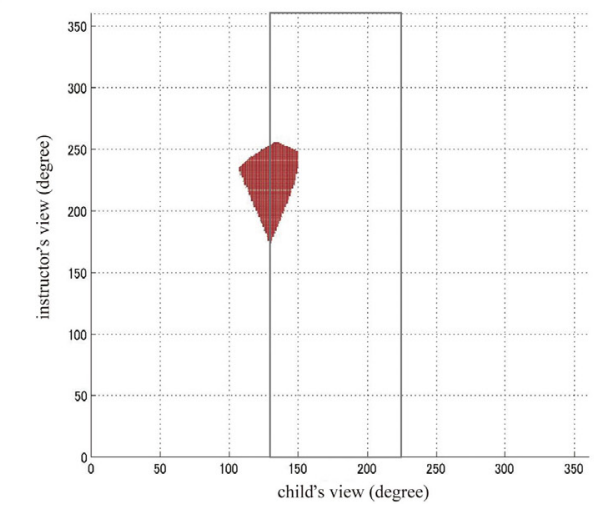

FIGURE 2 | Illustrations of the two-dimensional motion capture system and results of pre-helping behavior. (A) Children and instructors wore a hat with markers in different colors. (B) Video frame of session recorded by digital video cameras installed on the celling. (C) Positional information of a child and his instructor on a two-dimensional surface; an angle of $180^{\circ}$ indicates that the child was in front of the instructor. (D) Example of Gaussian distribution representing the spatial profile of a child's attention before helping behavior. The black arrow indicates the range of $x$-axis (in degrees) where the instructor was in front of the child, and the white arrow indicates the range of $y$-axis (in degrees) where the child was in front of the instructor. (E) Difference in pre-helping behavior between ASD (pre-baseline period) and typically developing children. In the region colored in red, children with ASD showed a significantly greater value of frequency compared to typically developing children $(\alpha=0.067)$. The area indicated by the gray rectangle indicates the range of $x$-axis (in degrees) where the instructor was in front of the child. The children with ASD looked straight at their instructor more often than did the typically developing children.

location and $y$ be the relative location of the child with respect to the instructor's location. In a given video frame, $x$ and $y$ were recorded as $x_{t}$ and $y_{t}$ (in degrees, $t=$ time), respectively, in the following ranges:

$$
0 \leq x_{t} \leq 360 ; 0 \leq y_{t} \leq 360 .
$$

When the child (or instructor) was in front of the instructor (or child), $x_{t}$ (or $y_{t}$ ) became $180^{\circ}$ (Figure $2 \mathrm{C}$ ).
Subsequently, this positional information was used to evaluate the state of the children's attention before the helping behavior. Based on the notion that human attention to space has a gradient, rather than a spotlight distribution (Mangun and Hillyard, 1988), we adopted a Gaussian distribution to represent the spatial profile of each child's attention during a given attempt, based on the positional relationship with their instructor. Because a peak 
of the Gaussian function appears at the origin of the coordinate system $(0,0)$, we transformed the $x y$ coordinate system into a $x^{\prime} y^{\prime}$ coordinate system, whose origin corresponded to $x_{t}$ and $y_{t}$ in the original system $(x y)$; therefore, we have

$$
\begin{aligned}
& x^{\prime}=x-x_{t}, \\
& y^{\prime}=y-y_{t} .
\end{aligned}
$$

For a given set of $x^{\prime}$ and $y,{ }^{\prime}$ the unnormalized two-dimensional Gaussian function for each video frame was defined as follows, where $\sigma=20$ taking the central visual angle of children into consideration:

$$
f(x, y)=\exp \left\{-\frac{\left(x^{\prime 2}+y^{\prime 2}\right)}{2 \sigma^{2}}\right\}
$$

The obtained Gaussian functions were then summed across all video frames over the period of a given attempt (10 s, Figure 2D). The amplitude of head movement is proportional to the amplitude of gaze shift amplitude for capturing visual targets in human (e.g., Barnes, 1979; Fang et al., 2015). Thus, these analyses enabled us to evaluate where and to what extent children directed their attention to their instructors before they performed helping behaviors. These analyses procedure were done by the Kinema tracer (Kissei Comtec Co., Ltd., Japan) and Matlab 7.8 (MathWorks, Japan).

\section{Data Analyses}

First, for the children with ASD, the level of prompt and number of attempts required in each session were examined to assess changes in helping behavior over the intervention. Second, we compared the ASD and typically developing children with respect to prompts and attempts required during the pre-baseline period (ASD children) and the experimental session (typically developing children). Regarding the pre-helping behavior, we divided the summed Gaussian distribution of the positional relationships by the number of video frames for a given attempt, due to the unequal numbers of frames, and subjected them to a $U$ test $(\alpha=0.067)$. Finally, the mean Gaussian distribution for children with ASD in the pre-baseline and post-baseline periods was contrasted to examine the intervention-related changes in pre-helping behavior. All analyses were done using Matlab 7.8 (MathWorks, Japan).

\section{RESULTS}

\section{Appearance of Helping Behavior in Children with ASD}

As shown in Figure 1B, both children with ASD became able to help their instructor with less-explicit and fewer prompts after the intervention. For Child A, the majority of his helping behavior appeared after level-4 prompts from pre-baseline to midterm baseline periods. In instruction period 2, he was able to perform the helping behavior with lower level prompts, and these were observed even without verbal stimulation in the post-baseline period. On the other hand, Child B was already able to perform the helping behavior in response to level-2 prompts during the pre-baseline period. In instruction period 1, he became able to respond to level-1 prompts, and this was observed constantly thereafter.

\section{Comparison of Typically Developing Children and Children with ASD}

All of the typically developing children performed the helping behavior after the first attempt of level-2 prompts. Compared to this result, the children with ASD were shown to require more explicit (i.e., level-4 prompt for Child A) and/or a greater number of attempts (i.e., third attempt of level-2 prompts for Child B), before the intervention or in the pre-baseline period. Regarding pre-helping behavior with respect to prompts/attempts, the children with ASD looked straight at their instructor significantly more often than did the typically developing children, as reflected by greater values of frequency within the region around $180^{\circ}$ (i.e., the instructor being in front of the child, $\alpha=0.067$, Figure 2E).

\section{Changes in Pre-Helping Behavior after the Intervention}

A comparison of pre-helping behavior before and after SST revealed an increase in the frequency of looking straight at the helpee in both children with ASD, as reflected by greater values of frequency within the region around $180^{\circ}$ (i.e., the instructor being in front of the child) after SST (Figure 3). In particular, Child B did so even when the helpee (i.e., his instructor) was not facing him.

\section{DISCUSSION}

The present SST targeting a helping behavior made it possible for two children with ASD to perform this behavior with fewer and less-explicit social stimulation. More specifically, after the intervention, the children with ASD could notice that their instructor was in need and offered help based on his/her nonverbal behavior (i.e., looking around for something). This result is consistent with previous studies that showed the effectiveness of a similar intervention in shaping helping behaviors (Matsuoka et al., 1999; Matsuoka and Noro, 2001; Suto, 2006, 2008; Suto and Oishi, 2007). Helping behaviors are considered to be effective in building relationships with others, whether spontaneous or motivated (Harris et al., 1990), and may increase the opportunities to receive social praise, such as appreciation from helpees (Matsuoka et al., 1999). Therefore, shaping appropriate helping behaviors in children with ASD may increase their chances of successful social interactions and facilitate interpersonal communication.

Moreover, the present study revealed characteristics of prehelping behaviors in children with ASD, which have rarely been addressed in previous studies using overhead-motion track studies due to technical difficulties. In the present research, children with ASD looked straight at the helpees before they offered help significantly more often than did typically developing children. This suggests that children with ASD must "observe" persons in need, while a "glance" at them may be enough for typically developing 

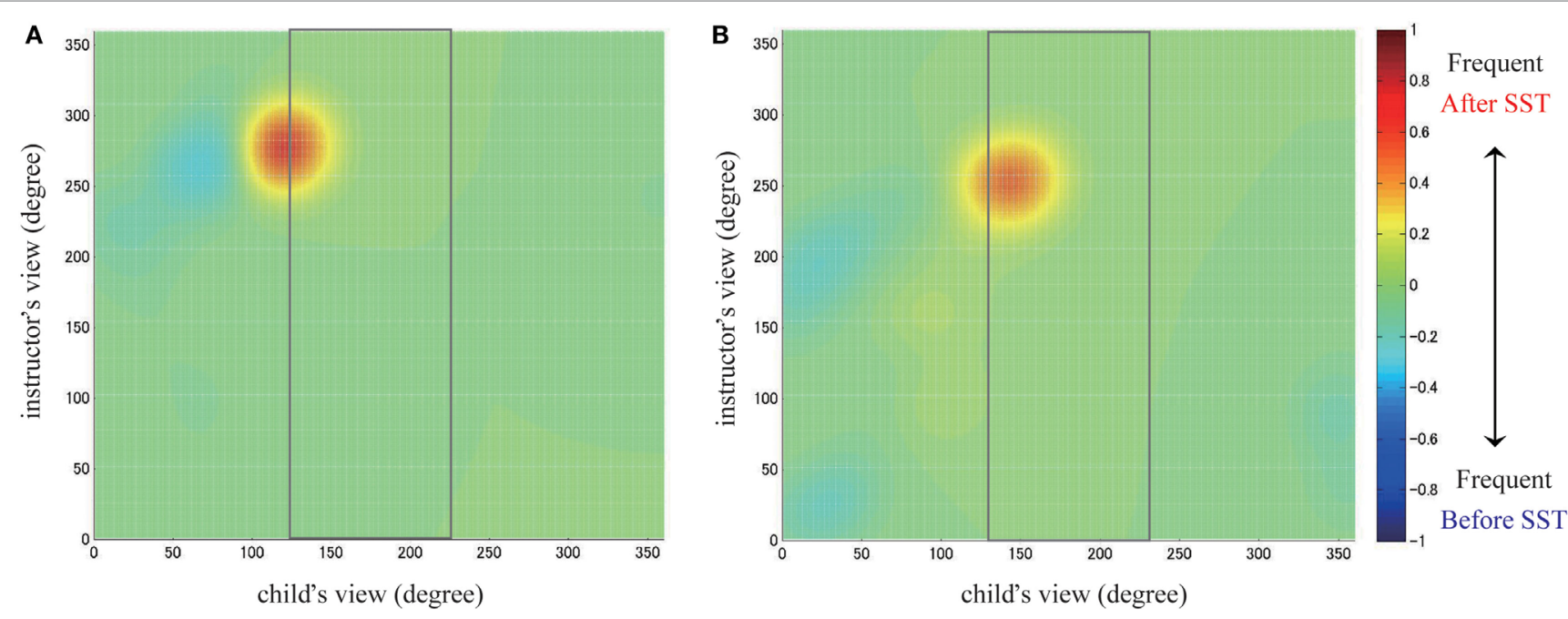

FIGURE 3 | Pre-post comparisons of pre-helping behavior in children with ASD (difference map). (A) Child A. (B) Child B. Brighter colors (toward red) indicate that values of frequency in that region increased after the intervention. The area indicated by the gray rectangle indicates the range of $x$-axis (in degrees) where the instructor was in front of the child. Both children looked straight at their instructor more often after SST.

children. Based on the model by Eisenberg (1986), attention to the other's need may be weaker in children with ASD and thus require careful observation, while typically developing children may instantly perceive such persons so that helping behaviors occur more easily. Spontaneous attention to social stimuli is considered to involve experiences of socialization, social cognitive development, and emotional understanding (Sugimura, 2009), and, in fact, difficulties in emotional understanding have been suggested as a cause of scarce helping behaviors in ASD (Bacon et al., 1998; Downs and Smith, 2004; Liebal et al., 2008). However, the main reason for the scarcity of helping behaviors in children with ASD may not only be low emotional understanding, but weaker attention to the other's need, leading to a decrease in such behaviors.

Over the course of the present intervention, improvement in helping behavior was associated with an increase in the frequency of looking straight at helpees before performing the helping behavior. This finding suggests that children with ASD can perform helping behaviors without explicit stimulation if they can successfully continue to observe the helpees for long time. The increments of "observation" time might give the children with ASD more opportunities to understand the situations and mental states of them. While classical interventions based on operant conditioning principles usually involve repetitive practice to achieve the acquisition of target behaviors (e.g., Frankel et al., 1987), the present study used model movies with follow-up questions. This procedure may have facilitated the children's understanding of the situations and mental states of persons in need, along with the imitation of appropriate helping behaviors. Such understanding may in turn have increased helping behavior in response to less-explicit social stimuli after SST.

Finally, it must be noted that the findings of this educational case study have several limitations. First, helping and pre-helping behaviors in the present study were directed to a particular person (i.e., an instructor working in a fixed pair). In previous studies, familiarity with a helpee and maturity in the relationship with him/her have been shown to strongly affect motivational factors (Eisenberg, 1986; Sugimura, 2009). Although the setting of the present study is necessary when considering the characteristics of ASD, an increase in children's familiarity and improvement in the relationship with their instructor may account for the present results. Therefore, examinations of helping and pre-helping behaviors directed to unfamiliar persons may be necessary in future studies to examine general improvements in helping behaviors. Second, as another problem of generalization, we did not assess other kinds of helping behaviors other than the target behavior (i.e., lending their items to their instructor). Moreover, the present study did not set follow-up periods after the intervention (e.g., 6 and 12 months) and the maintenance of behavior improvement remains unclear. Both problems of generalization and maintenance have been discussed for long time in the research field of educational intervention (e.g., Koegel and Rincover, 1977). We should address these basic problems in the coming researches. Third, the present finding must be further confirmed by involving larger numbers of children in randomized clinical trials. Although we adopted an intensive 1-month intervention to minimize the influence of natural development, this and other factors, such as emotional and cognitive states, must be more strictly controlled in future studies to draw stronger conclusions regarding the characteristics of social interactions in children with ASD and appropriate interventional approaches. These limitations can be mainly caused by experimental designs, small sample size, and lacks of control subjects, and further studies are expected to fix the limitations and generalize the present preliminary findings to other children with ASD. 


\section{ETHICS STATEMENT}

This study was carried out in accordance with the recommendations of "Ethical Guidelines for Medical and Health Research Involving Human Subjects; the ethics committee at the National Center of Neurology and Psychiatry (Protocol no. 22-4-JI14)" with written informed consent from all subjects. All subjects gave written informed consent in accordance with the Declaration of Helsinki. The protocol was approved by "the ethics committee at the National Center of Neurology and Psychiatry (Protocol no. 22-4-JI14)."

\section{AUTHOR CONTRIBUTIONS}

YK contributed to the design of the study and the data collection, analyzed and interpreted the data, drafted the manuscript, approved the final version of the manuscript, and agreed to be accountable to all aspects of this work. AG, RS, TG, and MI contributed to the design of the study and the data collection, drafted the manuscript, approved the final version of the manuscript, and

\section{REFERENCES}

American Psychiatric Association. (2013). Diagnostic and Statistical Manual of Mental Disorders, 5th Edn. Arlington, VA: American Psychiatric Association.

Bacon, A. L., Fein, D., Morris, R., Waterhouse, L., and Allen, D. (1998). The response of autistic children to the distress of others. J. Autism Dev. Disord. 28, 129-142. doi:10.1023/A:1026040615628

Barnes, G. R. (1979). Vestibulo-ocular function during coordinated head and eye movements to acquire visual targets. J. Physiol. 287, 127-147. doi:10.1113/ jphysiol.1979.sp012650

Carter, A. S., Davis, N. O., Klin, A., and Volkmar, F. R. (2005). "Social development in autism," in Handbook of Autism and Pervasive Developmental Disorders, eds F. R. Volkmar, R. Paul, A. Klin, and D. J. Cohen (New York, NY: Wiley), 312-334.

Downs, A., and Smith, T. (2004). Emotional understanding, cooperation, and social behavior in high-functioning children with autism. J. Autism Dev. Disord. 24, 625-635. doi:10.1007/s10803-004-5284-0

Eisenberg, N. (1982). The Development of Prosocial Behavior. London: Academic Press, Inc.

Eisenberg, N. (1986). Altruistic Emotion, Cognition, and Behavior. Hillsdale, NJ: Lawrence Erlbaum Associates.

Fang, Y., Nakashima, R., Matsumiya, K., Kuriki, I., and Shioiri, S. (2015). Eyehead coordination for visual cognitive processing. PLoS ONE 10:e0121035. doi:10.1371/journal.pone.0121035

Frankel, R. M., Leary, M., and Kilman, B. (1987). "Building social skills through pragmatic analysis: assessment and treatment implications for children with autism," in Handbook of Autism and Pervasive Developmental Disorders, eds D. J. Cohen, A. M. Donnellan, and R. Paul (Chichester: John Wiley \& Sons), 333-359.

Harris, S., Handleman, J. S., and Alessandri, M. (1990). Teaching youth with autism to offer assistance. J. Appl. Behav. Anal. 20, 297-305. doi:10.1901/ jaba.1990.23-297

Koegel, R., and Rincover, A. (1977). Research on the difference between generalization and maintenance in extra-therapy responding. J. Appl. Behav. Anal. 10, 1-12. doi:10.1901/jaba.1977.10-1

Liebal, K., Colombi, C., Rogers, S. J., Warneken, F., and Tomasello, M. (2008). Helping and cooperation in children with autism. J. Autism Dev. Disord. 38, 224-238. doi:10.1007/s10803-007-0381-5

Mangun, G. R., and Hillyard, S. A. (1988). Spatial gradients of visual attention: behavioral and electrophysiological evidence. Electroencephalogr. Clin. Neurophysiol. 70, 417-428. doi:10.1016/0013-4694(88)90019-3 agreed to be accountable to all aspects of this work. YO analyzed and interpreted the data, drafted the manuscript, approved the final version of the manuscript, and agreed to be accountable to all aspects of this work.

\section{ACKNOWLEDGMENTS}

We would like to thank Professor Toshihide Koike (Tokyo Gakugei University, Japan), Ms. Aya Kumazawa, Ms. Keiko Kosugi, and Ms. Tomoko Abe for their faithful assistance.

\section{FUNDING}

This work was supported in part by a Grant-in-Aid for Young Scientists (B) (grant number 26780524 to YK); Intramural Research Grant for Neurological and Psychiatric Disorders of NCNP (grant number 29-6 to YK and MI); Health Labour Sciences Research Grant (grant number H28-Kankaku-Ippan-001 to YK and MI).

Matsuoka, K., and Noro, F. (2001). Research on the formation of mutual helping behaviors in persons with developmental disabilities--preliminary examination using the framework of conditional distinction. Bull. Disabil. Sci. 25, $1-12$.

Matsuoka, K., Noro, F., and Kobayashi, S. (1999). Conditions for establishing helping behavior in an adolescents with autism. Jpn. J. Spec. Educ. 37, 51-58. doi:10.6033/tokkyou.37.51

Sally, D., and Hill, E. (2006). The development of interpersonal strategy: autism, theory-of-mind, cooperation, and fairness. J. Econ. Psychol. 27, 73-97. doi:10.1016/j.joep.2005.06.015

Sugimura, R. (2009). Trends in studies of prosocial behavior of children with developmental disorders--centrally focusing on pervasive developmental disorders. Annu. Bull. Grad. Sch. Educ. Tohoku Univ. 57, 239-254.

Suto, K. (2006). On the conditions for establishing helping behavior in children with autism--suggestions from the experience of receiving help. Annu. Rep. Psychol. Meisei Univ. 24, 1-15.

Suto, K. (2008). An examination of the conditions for creating helping behavior in children with autism--through observational responses of assistants. Jpn. J. Educ. Psychol. 56, 268-277. doi:10.5926/jjep1953.56.2_268

Suto, K., and Oishi, K. (2007). On the conditions for establishing helping behavior in children with autism--suggestions from emotional stimuli. Rikkyo Psychol. Res. 49, 73-84.

Tsujii, M. (2004). Children with Pervasive Developmental Disorders--To Understand High Functioning Autism and Asperger's Syndrome. Tokyo: Brain Publishing.

Wincze, J. P., Leitenberg, H., and Agras, W. S. (1972). The effects of token reinforcement and feedback on the delusional verbal behavior of chronic paranoid schizophrenics. J. Appl. Behav. Anal. 5, 247-262. doi:10.1901/jaba.1972. 5-247

Conflict of Interest Statement: The authors declare that the research was conducted in the absence of any commercial or financial relationships that could be construed as a potential conflict of interest.

Copyright $\odot 2017$ Kita, Gunji, Sakuma, Goto, Okumura and Inagaki. This is an open-access article distributed under the terms of the Creative Commons Attribution License (CC BY). The use, distribution or reproduction in other forums is permitted, provided the original author(s) or licensor are credited and that the original publication in this journal is cited, in accordance with accepted academic practice. No use, distribution or reproduction is permitted which does not comply with these terms. 